\title{
Simulation of the Reflectivity Properties of Microstructured Titanium Surface by Ray Tracing Method
}

\author{
Tamás CSIZMADIA ${ }^{* 1,2}$, Miklós ERDÉLYI $^{* 1}$, Tomi SMAUSZ $^{* 3}$, Tibor NOVÁK ${ }^{* 1}$, Béla HOPP ${ }^{* 1}$ \\ ${ }^{* 1}$ Department of Optics and Quantum Electronics, University of Szeged, H-6720 Szeged, Dóm tér 9, Hungary \\ ${ }^{* 2}$ Attosecond Light Pulse Source, ELI-Hu Nkft, Szeged H-6720, Dugonics tér 13, Hungary \\ E-mail:Tamas.Csizmadia@eli-alps.hu \\ ${ }^{* 3}$ MTA-SZTE Research Group on Photoacoustic Spectroscopy, University of Szeged, 6720 Szeged, Dóm tér 9, Hungary
}

\begin{abstract}
Improving light trapping properties of metal surfaces by pulsed laser irradiation based techniques is a popular research field, which has numerous promising practical applications and technological importance. Albeit several studies have been published on the control of the reflectivity properties by means of micro- and nanostructures, there are still open questions regarding the reflectivity reduction mechanism of the processed metal surface. In this work, the calculation of the reflectivity of a simulated titanium surface having microstructures prepared by femtosecond laser irradiation is demonstrated using the ray tracing method. The morphological features of the modeled surface were adopted from the paper of Nayak et al. using their SEM pictures of femtosecond laser irradiated Ti surface [1]. The simulated total reflectivity value of the surface was compared to the experimental data and good agreement was found between them. This indicates that the reflectivity properties of certain microstructured surfaces can be appropriately described by geometrical optics approaches and the ray tracing method can be a proper technique for calculating the total reflectivity value of such surfaces.
\end{abstract}

DOI: $10.2961 / \mathrm{jlmn} .2015 .02 .0019$

Keywords: titanium, microstructures, simulation, ray tracing, reflectivity, darkening, laser

\section{Introduction}

Micro- and nanomachining of different surfaces has significant practical applications in wide range of research fields, for example in controlling the wetting properties of the surface $[2,3]$, in modifying the static and kinetic friction properties of surfaces for tribological applications $[4,5]$, in improving cell growth [6], and in creating non-reflective surfaces for optical and photovoltaic applications $[7,8]$. The optical properties of microstructured surfaces can be calculated by ray tracing method in case of having formations that are characteristically larger than the wavelength of the illuminating light [9-12]. Hua et al. investigated the light-trapping effect of different silicon structures including close-packed triangular, rectangular and hexangular pyramid structures, close-packed cone structures and square array cone structures for optimizing the texture shape on front surface of solar cell [13]. The anti-reflection efficiency of inverted pyramidal structures with tips in their center was studied numerically and experimentally for anti-reflection applications and good agreement was found between the measured and the numerically derived reflectance values [14]. In this work, a ray tracing calculation of the reflectivity of titanium surface having microstructures prepared by femtosecond laser irradiation is demonstrated. The discussion of the opportunities and limits of the applied model and the simulation method is also included.

In earlier experiments Nayak et al. produced self-organized cone-like microstructures on a bulk titanium surface [1]. The geometrical parameters of these structures (height and inter-cone distance) were investigated as a function of the applied laser fluence and the number of the irradiating laser pulses. The processing light source was a Ti-Sapphire laser system $(\lambda=800 \mathrm{~nm}$,
$F W H M=130 \mathrm{fs})$. Figure 1 shows the formed cone-like microstructures when increasing the laser fluences $(F=$ $\left.0.3-1.2 \mathrm{~J} / \mathrm{cm}^{2}\right)$, while the pulse number was kept at a constant value $(N=450)$. The heights of the cones and the inter-cone distance between them were determined by atomic force microscope (AFM) measurements. Such experiments revealed how these geometrical properties depend on the laser processing parameters (applied fluence and pulse number). It was shown that the reflectivity of Ti surfaces can be controlled and reduced to practically zero, opening up the possibilities for their application in the field of photovoltaics. The main reason behind the observed reflectivity decrease is that the incident light reflects several times among the surface microstructures before it finally exits from the surface. This suggests that the dependence of the reflectivity spectrum on the pulse number is caused by the various geometrical sizes and forms of the laser prepared surface structures. To investigate this hypothesis the effect of the prepared surface structures on surface reflectivity was simulated by TracePro, a commercially available ray tracing software.

\section{Applied methods and simulation results}

In the first step, a model of the laser prepared surface was implemented into the TracePro software. For that, the reflectivity values of the smooth Ti surface were recorded in a new catalog according to Table 1.

Cones with an average height and diameter of $32 \mu \mathrm{m}$ and $22 \mu \mathrm{m}$, respectively were placed on a rectangular cuboid in a square grid formation, where the side length of the grid was equal to the diameter of the placed cones (Figure 2). The values for the cone height and the diameter of the cone base were selected based on previous AFM measurements [1]. 

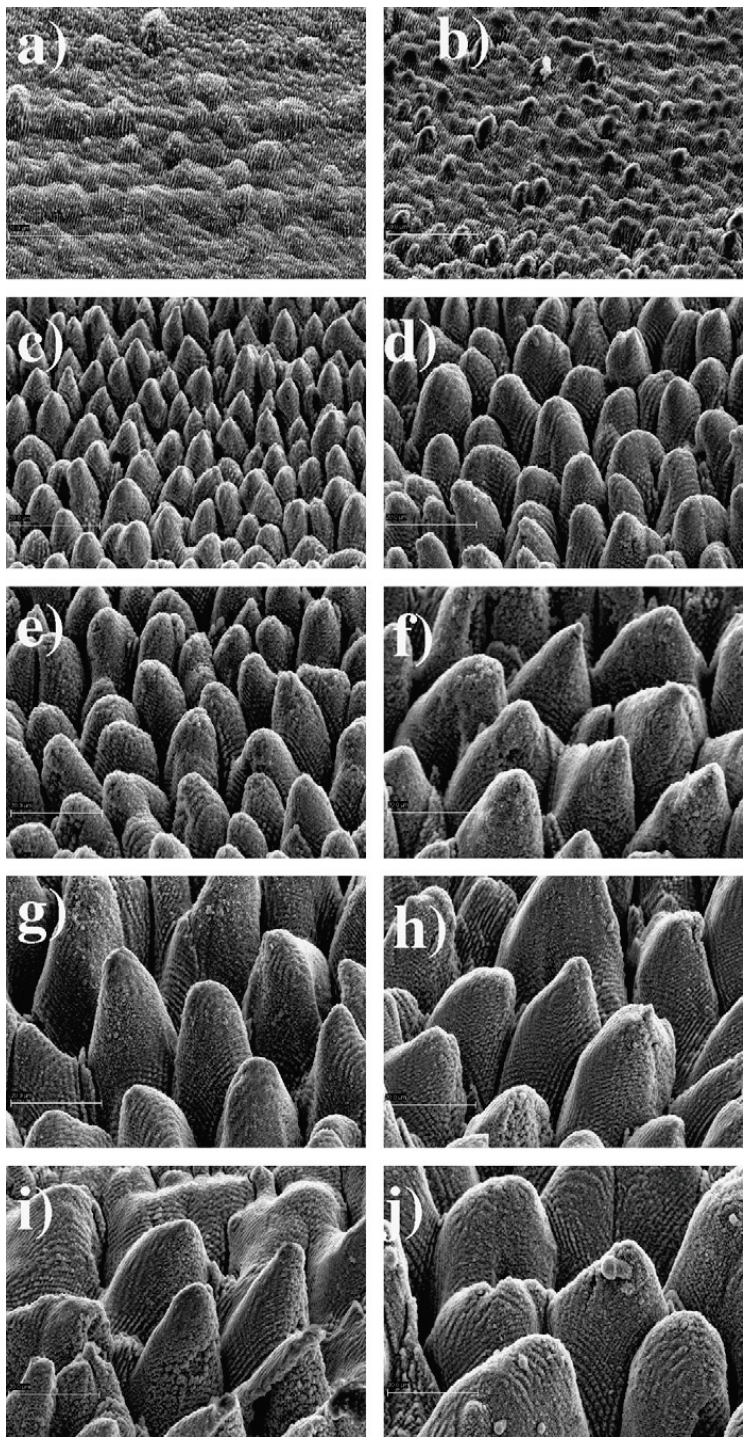

Fig. 1: Formation of conical surface structures with increasing laser fluence in $\mathrm{J} / \mathrm{cm}^{2}$ for (a) 0.3 (b) 0.4 (c) 0.5 (d) 0.6 (e) 0.7 (f) 0.8 (g) 0.9 (h) 1 (i) 1.1 (j) 1.2.The number of laser shots for all cases was 450 . Scale bar is $20 \mu \mathrm{m}$. From Nayak, 2010 [1].

It is worth noting here that in the applied reference work [1] both the reflectivity spectra and the geometrical parameters of the microstructures were given only for a single laser processing setup $(F=1$ $\mathrm{J} / \mathrm{cm}^{2}$ and $\left.N=500\right)$. Therefore the geometric parameters at that fluence and pulse number values had to be selected for further comparison of the calculated and the measured reflectivities. For evaluating the results, it was necessary to surround the created geometry with a surface having $100 \%$ transmission, where the total amount of power reflected from the Ti surface can be integrated. The ratio of the integrated reflected power and the total incident power provides the calculated reflectivity value. The applied plane light source was parallel, while the emitted unpolarized rays were perpendicular to the base of the cones. Initially, the wavelength of the rays was $600 \mathrm{~nm}$, and the area of the plane light source matched to the area of the applied geometry (Figure 2).

Table 1 Reflectivity values of the smooth Ti surface

\begin{tabular}{|c|c|c|c|}
\hline $\begin{array}{c}\text { Incident angle } \\
\left({ }^{\circ}\right)\end{array}$ & $\lambda=600 \mathrm{~nm}$ & $\lambda=750 \mathrm{~nm}$ & $\lambda=900 \mathrm{~nm}$ \\
\hline 0 & 0.5231 & 0.5591 & 0.54834 \\
\hline 10 & 0.52309 & 0.55909 & 0.54833 \\
\hline 20 & 0.52298 & 0.5589 & 0.54812 \\
\hline 30 & 0.52247 & 0.55803 & 0.5472 \\
\hline 40 & 0.52113 & 0.5556 & 0.54454 \\
\hline 50 & 0.51868 & 0.55031 & 0.53859 \\
\hline 60 & 0.51644 & 0.54125 & 0.52769 \\
\hline 70 & 0.52435 & 0.53316 & 0.51449 \\
\hline 75 & 0.54442 & 0.53899 & 0.51511 \\
\hline 80 & 0.59595 & 0.57181 & 0.54026 \\
\hline 85 & 0.71995 & 0.68197 & 0.64493 \\
\hline 87 & 0.80684 & 0.77275 & 0.74004 \\
\hline 89 & 0.92587 & 0.9093 & 0.89294 \\
\hline 90 & 1 & 1 & 1 \\
\hline & & & \\
\hline
\end{tabular}

Table 1: Reflectivities of a smooth Ti surface at three wavelengths $(\lambda=600,750$ and $900 \mathrm{~nm})$ and at different incident angles. [15]

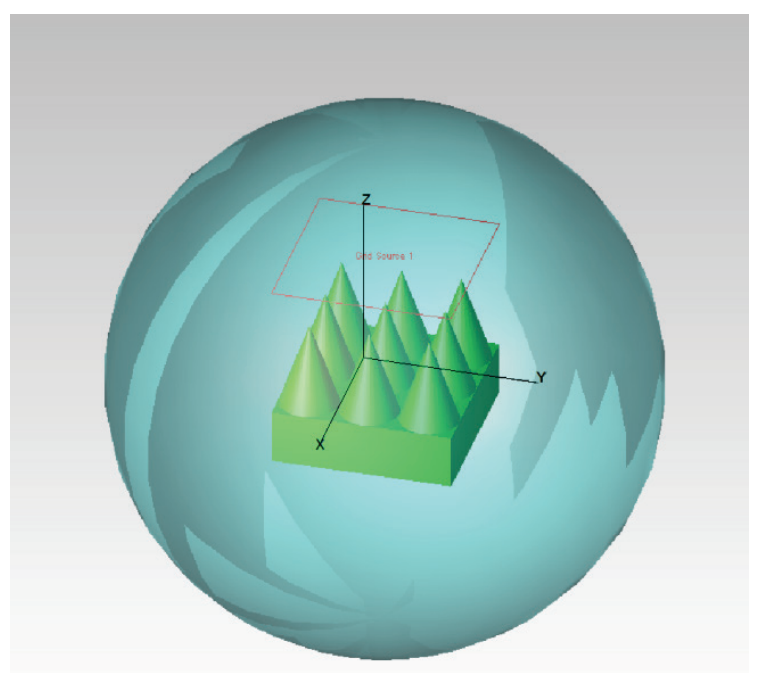

Fig. 2: The constructed geometry for the ray tracing simulation (using $3 \times 3$ cones). The plane light source is marked with the red square above the green geometry. The whole setup was surrounded by a perfect transmitter sphere, on which the reflected rays were summarized. 
The ray tracing calculation time depends on the size of the modeled surface: the larger the surface, the more time consuming the simulation is. However, in the real reflectivity measurements a very large number of cones are illuminated. Reduction of the number of illuminated cones $(N)$ shortens the calculation time but affects the simulation results too. To find the optimum size in the model, different geometries $(N=4,9,16,36$ and 100) were designed and the ray tracing simulation were evaluated on every one of them.

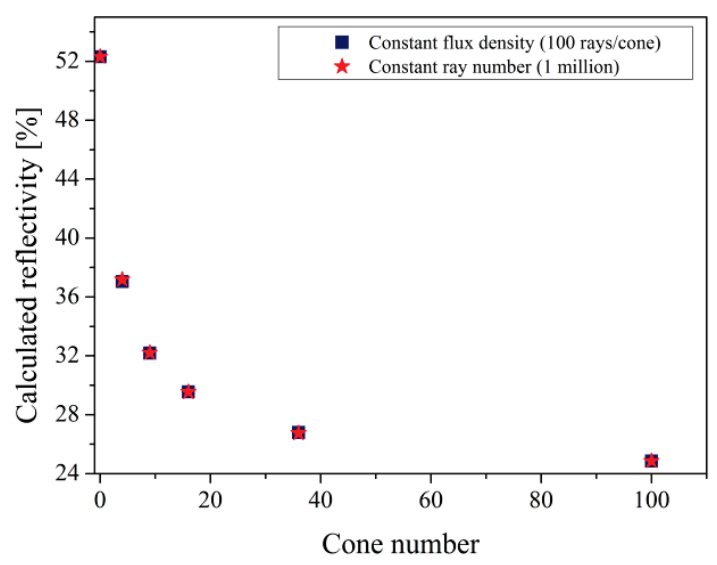

Fig. 3: The calculated reflectivity as the function of the number of cones used in the built geometry model. The wavelength of the irradiating rays was $600 \mathrm{~nm}$.

In Figure 3 the dependence of the calculated reflectivity on the cone number can be seen. The calculations were carried out in two different ways: in the first one the number of the incident rays was varied with the number of the cones, so the flux density was invariant (100 rays/cone), while in the second case the number of the incident rays was kept in a constant value of $10^{6}$. It can be seen in Figure 3 that the number of the applied rays does not considerably influence the calculated reflectivity in the investigated range. However, the number of the cones has a significant effect: the calculated reflectivity reduces from the original $52.31 \%$ to $37 \%$ already for 4 applied cones, and it decreases even more with the ascendant cone number, and slowly approaches approx. $24 \%$ reflectivity value. Based on this result, the geometry including 100 cones was applied in the further calculations.

Another important parameter, which heavily determines the calculation time is the number of the emitted rays from the light source. Therefore it is important to find out the minimum ray number required to get accurate reflectivity values. Figure 4 reveals that reflectivity increases with the increasing ray number up to $10^{5}$ rays, after which saturation can be observed.

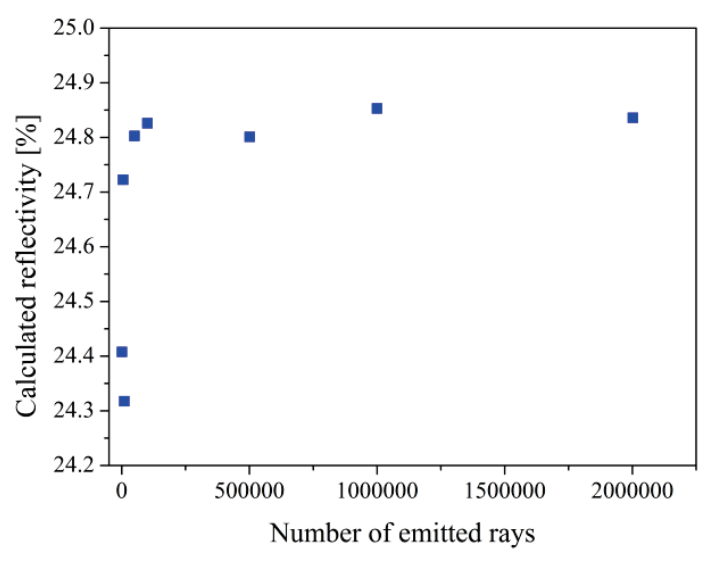

Fig. 4: The dependence of the calculated reflectivity on the number of the applied rays.

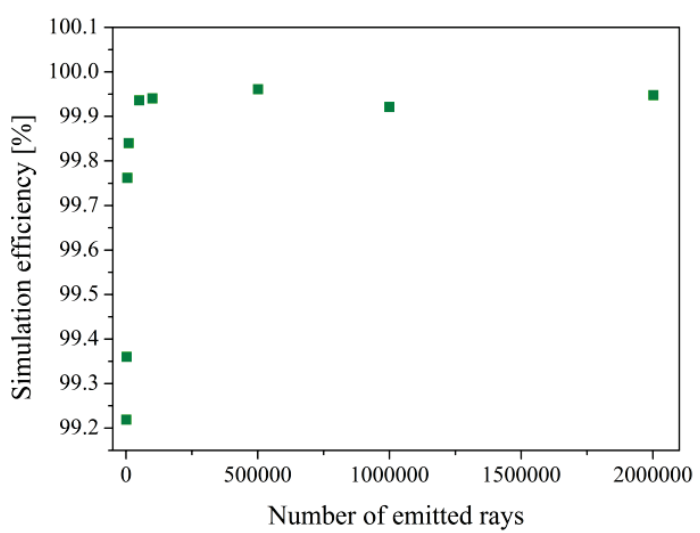

Fig. 5: The dependence of the simulation efficiency on the number of the applied rays.

The rays which cross the edge of a structure cannot be traced, therefore the energy transported by them has been considered as lost. This artifact causes the calculated reflection value to be lower than the expected one. The ratio of the exited rays through the surrounding margin and the emitting rays from the light source was denominated as the simulation efficiency. The relationship between the simulation efficiency and the number of the applied rays follows a similar trend as was plotted in Figure 4, which indicates that the smaller reflectivities at lower ray numbers are due to the rays, which could not be tracked during the calculation process. To achieve more than $99.9 \%$ accuracy in the calculated reflectivity values, more than $10^{5}$ rays was applied in subsequent calculations.

Using 100 cones and $10^{5}$ rays in the simulation, the reflectivity was found to be $24.8 \%$. In the next step, the alignment of the cones on the rectangular cuboid was modified, while the inter-cone distance remained constant $22 \mu \mathrm{m}$. The cones were relocated from the square grid to the rhombus grid arrangement having a side length of $22 \mu \mathrm{m}$, which provides better covering of the surface (Figure 6). The calculated reflectivity for this modified rhombus grid geometry was $19.88 \%$, which is 
5 percentage points lower than it was in case of the previously used square grid geometry.

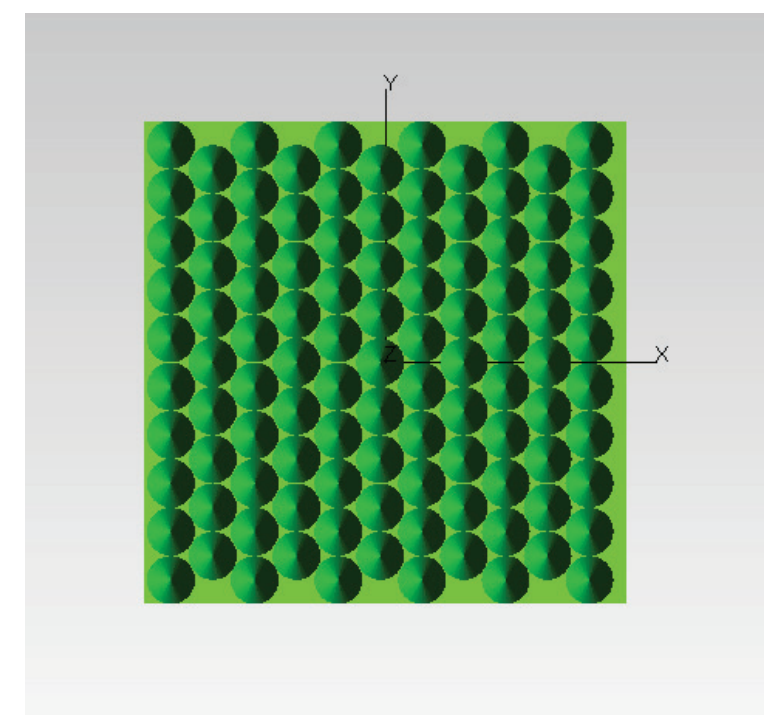

Fig. 6: Top view of the modified geometry, which contains 105 cones in a rhombus grid arrangement.

In real reflectivity measurements, the area of the structured surface is significantly larger than the illuminated area; therefore it is important to find out what happens with the calculated reflectivity, when the size of the light source is reduced. In this case, the number of the irradiated cones is also decreased, but the rays reaching the surface in the outer regions do not exit after one reflection, which better corresponds to the real case. Hence the error introduced by the finite size of the modeled geometry can be reduced. Figure 7 shows that the calculated reflectivity fluctuates around $13.5 \%$ below $80 \%$ illumination and then starts to increase with the size of the illuminated area. The number of the emitted rays was constant $10^{5}$ during this simulation.

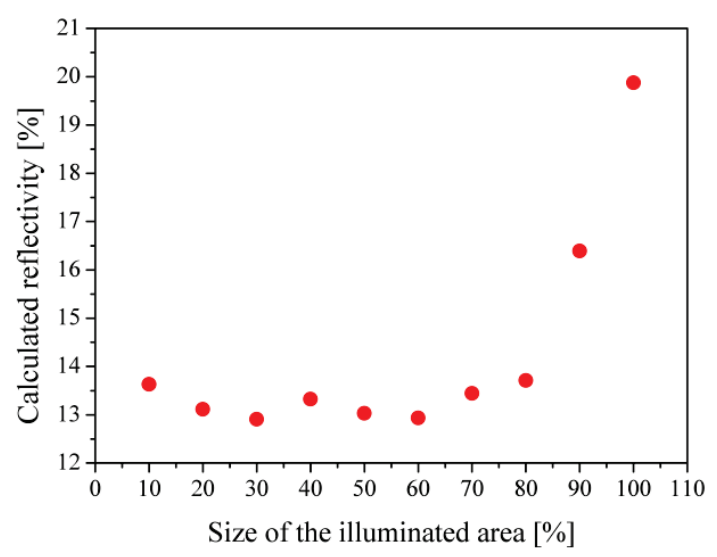

Fig. 7: The calculated reflectivity as a function of the illuminated area, which equals the size of the plane light source.

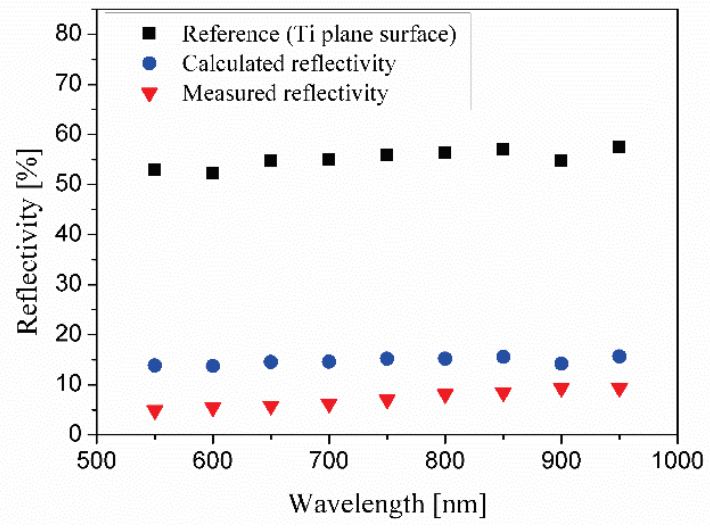

Fig. 8: Comparison of the calculated and the measured reflectivities of the structured $\mathrm{Ti}$ surface with that of smooth $\mathrm{Ti}$ plane reference surface as the function of the wavelength. The simulation efficiency was over $99.9 \%$.

Regarding the above mentioned observations, the final model was configured using the following parameters: 105 cones were placed in a rhombus grid arrangement to get the best covering on the rectangular cuboid surface. $80 \%$ of the structured geometry was illuminated with $10^{5}$ rays. Figure 8 shows the result of the simulation at nine distinct wavelengths between 550 and $950 \mathrm{~nm}$. It can be seen in Figure 8, that the calculated reflectivity of the structured surface is quarter of the unstructured $\mathrm{Ti}$ plane surface reflectivity, which indicates that the multiple reflections among the overhanging surface structures plays a very important role in the reduction of the surface reflectivity.

\section{Conclusion}

In this work laser processed $\mathrm{Ti}$ surface was simulated in the TracePro software and the modified reflectivity of the patterned metal sample was calculated by ray tracing method on the basis of the experimentally determined surface topography. The used simulation model was optimized regarding the applied geometry (number of cones on the surface and their disposition) together with the simulation parameters (number of the emitted rays and the size of the illuminated area). The final setup contained 105 cones in a rhombus grid arrangement. The edges of the rhombus grid were $22 \mu \mathrm{m}$ long, which equaled the diameter of the cone base, while the height of the cones was $32 \mu \mathrm{m}$ according to the result of the AFM measurements in the cited paper. The calculated reflectivity of the structured surface is significantly lower than the reflectivity of the original flat Ti surface, which proves that the internal reflections, which are generated only by the changed morphology of the surface, have a considerable role in the drop of surface reflectivity. Our simulation shows that the main light trapping property of the microstructured Ti surface can be modeled by a geometrical optics approach; however, the reflectivity values produced by the model 
was 6.7 percentage points higher than the results of the real measurements, which requires further discussion.

The main differences between the structures of the real surface and the applied geometry is that the real structures are not regular cones on the one part, and on the other the arrangement of these cones is also irregular. It was proved previously that a slight change in the arrangement of the cones results in a significant change in the calculated reflectivity. When the cones were rebuilt from the square to the rhombus grid arrangement, the number of cones per area unit increased only by $5 \%$, but at the same time the calculated reflectivity decreased by $20 \%$. We believe that a structure having such small spaces between the cones as it is shown in Figure 1 would have even lower calculated reflectivity than our perfectly conical texture. This would put the measured and the calculated results closer to each other. Another interesting fact revealed in Figure 1 is that the laser prepared cones have different nanostructures on their surface. The size of these nanostructures is close to the wavelength of the illuminating light, therefore they scatter the incident light and reduce the reflectivity. This effect cannot be modeled by a geometric optics based method applicable for large feature size interfaces, although also takes part in the light trapping phenomena. Because of these scattering effects, the calculated reflectivity is only an upper estimation for the reflectivity of the real surfaces. Going beyond the limits of ray tracing a supercritical scattering approach was introduced by Riyopoulos et al. in order to treat the scattering effect caused by short feature scales on the structured surface [17].

It also has to be noted that during femtosecond irradiation of Ti in ambient air the chemical composition of the surface can be modified resulting the appearance of $\mathrm{TiO}_{2}$ and nonstoichiometric TiN films and nanoparticles on the surface [16]. This laser induced chemical modification was observed using 50 pulses and $250 \mathrm{~mJ} / \mathrm{cm}^{2}$ laser fluence in Ref. [16]. In the reference work [1] significantly higher fluence and pulse number values were used, therefore it is highly possible that different compounds of $\mathrm{Ti}$ were produced on the laser irradiated regions. The reflectivity of the chemically modified surface is lower than the reflectivity of pure $\mathrm{Ti}$ (for example $18.8 \%$ and $55.91 \%$ for $\mathrm{TiO}_{2}$ and $\mathrm{Ti}$ at 750 nm, respectively [15]), which could cause the measured reflectivity to be lower than the calculated one.

Figure 9 shows that in case of perpendicular illumination the main part of reflected intensity exits the surface with direct reflection, while the other part of the reflected intensity (about 30\%) exits by diffuse reflection. Such low- and diffusely reflecting metal surfaces can have interesting applications in the field of optoelectronics, photovoltaics, detectors and biomedicines in the coming future.

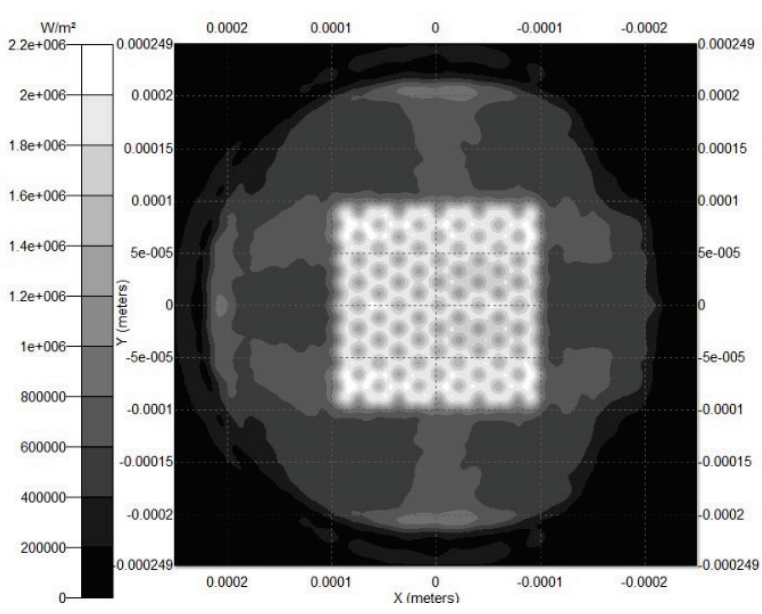

Figure 9: The spatial distribution of the reflected intensity around the modeled geometry. The intensity was measured on the spherical margin around the modeled geometry. The figure shows the 2-dimensional projection of this hemispherical boundary surface.

\section{Acknowledgements}

This work was partially supported by the European Union and co-financed by the European Social Fund through projects „Impulse lasers for use in materials science and biophotonics" (grant no.: TÁMOP4.2.2.A-11/1/KONV-2012-0060) and „Supercomputer, the national virtual lab" (TÁMOP-4.2.2.C-11/1/KONV2012-0010) and was realized in the frames of „National Excellence Program - Elaborating and operating an inland student and researcher personal support system convergence program" (TÁMOP 4.2.4. A/2-11-1-20120001). The authors thank Lambda Research Corporation for concession of the TracePro ${ }^{\circledR}$ software license used for the studies. M.E. is supported by the Marie Curie Integration Grant (PCIG13-GA-2013-618273) and the János Bolyai Research Scholarship of the Hungarian Academy of Sciences. The ELI-ALPS project (GOP1.1.1-12/B-2012-0001) is supported by the European Union and co-financed by the European Regional Development Fund.

\section{References}

1. B. K. Nayak and M. C. Gupta: Opt. Laser. Eng., 48, (2010) 940.

2. M. Hans, F. Müller, S. Grandthyll, S. Hüfner and F. Mücklich: App. Surf. Sci., 263, (2012) 416.

3. B. Wu, M. Zhou, J. Li, X. Ye, G. Li and L. Cai: App. Surf. Sci., 256, (2009) 61.

4. T. R. Jervis, M. Nastasi, Jr. A. J. Griffin, T. G. Zocco, T. N. Taylor and S. R. Foltyn: Surf. Coat Tech., 89, (1997) 158.

5. J. Li, D. Xiong, H. Wu, J. Huang, J. Dai and R. Tyagi: Tribol. Trans., 53, (2010) 195.

6. G. Pető, A. Karacs, Z. Pászti, L. Guczi, T. Divinyi and A. Joób: Appl. Surf. Sci., 186, (2002) 7 . 
7. B. Hopp, T. Smausz, T. Csizmadia, C. Vass, C. Tápai, B. Kiss, M. Ehrhardt, P. Lorenz and K. Zimmer: App. Phys. A, 113, (2013) 291.

8. H. Tao, J. Lin, Z. Hao, X. Gao, X. Song, C. Sun and X. Tan: App. Phys. Lett., 100, (2012) 201111.

9. Y. Cocheril and R. Vauzelle: PIER 75, (2007), 357.

10. Y. Han, X. Yu, D. Wang and D. Yang: J. Nanomater., 2013, (2013), 716012.

11. D. Bergström, J. Powell, and A. F. Kaplan: J. Appl. Phys 101, (2007), 113504.

12. D. Bergström, J. Powell, and A. F. Kaplan: J. Appl. Phys., 103, (2008), 103515.

13. X.-S. Hua, Y.-J. Zhang and H.-W. Wang: Sol. Energ. Mat. Sol. 94, (2010), 258.

14. H.-H. Cheng, Y.-Y. Chang, J.-Y. Chu, D.-Z. Lin, Y.-P. Chen, and J.-H. Li: Appl. Phys. Lett., 101, (2012), 141113.

15. Source of the data in Table 1: http://refractiveindex.info:I.D. Mash, G.P. Motulevich: JETP, 36, (1973), 516. Last access: February 20, 2015.

16. E. C. Landis, K. C. Phillips, E. F.Mazur, M. Cynthia: J. Appl. Phys., 112, (2012), 063108.

17. S. Riyopoulos, T. D. Moustakas and J. S. Cabalu: J. Appl. Phys., 102, (2007), 043111.

(Received: June 15, 2014, Accepted: April 13, 2015) 